\title{
RESONANCE IN ANISOTROPIC LAYERED MEDIA
}

\author{
Stephen P. Shipman, Aaron Welters \\ Louisiana State University, Department of Mathematics, Baton Rouge, LA 70803, USA \\ shipman@math.lsu.edu
}

\begin{abstract}
When a periodic anisotropic layered medium is interrupted by a slab of contrasting material, electromagnetic resonance can be manifest in a variety of ways. In one scenario, anisotropy allows for frequency intervals in which waves propagating perpendicular to the layers coexist with evanescent waves. This permits energy to be trapped within the slab at frequencies embedded in the continuous spectrum. These are unstable states that result in resonance under small perturbations. In another scenario, a stationary inflection point in the dispersion relation of an ambient magnetic anisotropic layered medium leads to unidirectional propagation and resonant interaction between Bloch waves and the contrasting layer. Analysis of energetic interactions between waves allows for a detailed understanding of these resonances.
\end{abstract}

\section{RESOnANCE In Slab Structures}

When a periodically layered medium is broken by a finite-width slab of a contrasting layered medium (Fig. 1), resonant interaction between an electromagnetic source field and the slab can result in anomalous scattering behavior. This study concerns resonance of a particular nature. We consider the situation in which the structure supports a harmonic electromagnetic field at a specific frequency in the absence of any source field originating from outside the slab (a source-free field). Such a field is dynamically decoupled from energy-transporting harmonic waves in the ambient medium. But when the parameters of the system are perturbed (structure, frequency, angle of incidence), waves in the ambient medium resonate with the slab, resulting in anomalous scattering.

This type of resonance requires that the ambient medium admit both evanescent and propagating waves at the same frequency and wavevector parallel to the layers, and it must therefore be anisotropic with respect to rotations in the plane of the layers. This is in contrast to Fabry-Perot resonance, which does not require a source-free field and occurs commonly in dielectric slabs surrounded by air. This paper examines the following two cases of resonant scattering which are elucidated by concrete calculations:

(A) Resonance with a guided mode. A source-free field is typically constructed by tuning the parameters of the system so that an evanescent field to the left of the slab $(z<0$ in Fig. 1) can be matched to an evanescent one to the right $(z>L)$, resulting in a bound state (with respect to the $z$-axis) that is physically a guided mode of the slab. In order for a perturbation of the system to excite resonant interaction between a guided mode and a propagating wave in the ambient medium, this medium must admit a propagating wave, implying that the frequency of the guided mode must be embedded in the continuum; in particular, this excludes purely isotropic layered materials. These guided modes are unstable with respect to generic perturbations, which result in sharp scattering anomalies such as sensitive dependence of the transmission coefficient to frequency.

(B) A unidirectional ambient medium. By alternating layers of an anisotropic dielectric and a magnetic medium possessing Faraday rotation, Figotin and Vitebskiy [2] have created a composite periodic medium with an asymmetric dispersion relation $\omega=W\left(k_{3}\right)$ relating frequency to the component $k_{3}$ of the Bloch wavevector $\mathbf{k}$ perpendicular to the layers. By tuning the parameters, they obtain a stationary cubic inflection point on the dispersion relation. At the frequency of this stationary point, the $4 \times 4$ transfer matrix across one period has a nontrivial Jordan canonical form with a Jordan block of size three. In particular, it has a degenerate eigenvalue and is nondiagonalizable. The Bloch wave corresponding to this eigenvalue has vanishing energy flux, and the sole propagating wave corresponds to the other eigenvalue, which is simple. The result is a medium that allows propagation of energy across the layers in only one direction. In nonresonant scattering, an incident field in the direction allowed by the medium excites a normal mode possessing unbounded linear growth in space. If the system supports a source-free field, this field is extended in space but its energy flux vanishes (although it may have a nonzero phase velocity), and it interacts resonantly with incident radiation when the system is perturbed.

Our analysis of electrodynamics in lossless layered anisotropic media is based on the transfer matrix method [1]. Let $\mathbb{R}^{3}$ be referred to variables $(x, y, z)$, with the $z$-axis running perpendicular to the layers (Fig. 1). One considers

(C)IEEE. This communication was supported by the European Science Foundation's NEWFOCUS Research Networking Program. 
electromagnetic fields of the form

$$
\mathbf{E}(x, y, z ; t)=\left\langle E_{1}(z), E_{2}(z), E_{3}(z)\right\rangle e^{i\left(k_{1} x+k_{2} y-\omega t\right)}, \quad \mathbf{H}(x, y, z ; t)=\left\langle H_{1}(z), H_{2}(z), H_{3}(z)\right\rangle e^{i\left(k_{1} x+k_{2} y-\omega t\right)},
$$

in which $\omega$ is the circular frequency and $\left(k_{1}, k_{2}\right)=\mathbf{k}_{\|}$is the wavevector parallel to the layers.

Denoting the tangential components of the field by a column vector

$$
\mathcal{F}(z)=\left[E_{1}(z), E_{2}(z), H_{1}(z), H_{2}(z)\right]^{T},
$$

the lossless time-harmonic Maxwell equations reduce to a $4 \times 4$ system of ODEs

$$
\frac{d}{d z} \mathcal{F}(z)=i \mathcal{A} \mathcal{F}(z)
$$

in which the matrix $\mathcal{A}=\mathcal{A}\left(z, \mathbf{k}_{\|}, \omega\right)$ is selfadjoint with respect to an indefinite sesquilinear energy-flux form $\left[\mathcal{F}_{1}, \mathcal{F}_{2}\right]$ that has a $2 \mathrm{D}$ negative-definite subspace and a $2 \mathrm{D}$ positive-definite subspace.

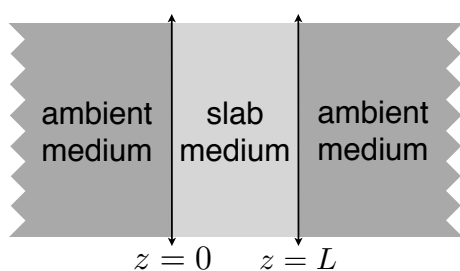

Figure 1. An infinite periodically layered anisotropic material is interrupted by a slab of length $L$ of a contrasting layered medium.

\section{Resonance With a Guided Mode}

Construction of a guided slab mode as described in case (A) above is possible even when both the ambient space and slab are homogeneous, as illustrated by the example that follows. The construction can be extended to genuinely layered ambient and slab media.

Let the electric and magnetic tensors $\epsilon^{(0)}$ and $\mu^{(0)}$ of the ambient space be

$$
\epsilon^{(0)}=\left[\begin{array}{ccc}
\epsilon_{1} & 0 & 0 \\
0 & \epsilon_{2} & 0 \\
0 & 0 & 1
\end{array}\right], \quad \mu^{(0)}=\left[\begin{array}{ccc}
\mu_{1} & 0 & 0 \\
0 & \mu_{2} & 0 \\
0 & 0 & 1
\end{array}\right] . \quad \text { (ambient space) }
$$

Let us consider fields that propagate parallel to the $x z$-plane, which means that $k_{2}=0$. One then computes that the propagation exponents $k_{3}$ in the $z$-direction are the four numbers $\left\{k_{3}^{0 e},-k_{3}^{0 e}, k_{3}^{0 p},-k_{3}^{0 p}\right\}$ given by

$$
k_{3}^{0 e}=\left[\epsilon_{1}\left(\frac{\omega^{2}}{c^{2}} \mu_{2}-k_{1}^{2}\right)\right]^{1 / 2}, \quad k_{3}^{0 p}=\left[\mu_{1}\left(\frac{\omega^{2}}{c^{2}} \epsilon_{2}-k_{1}^{2}\right)\right]^{1 / 2} .
$$

Their associated eigenspaces are given by the relations

$$
\begin{aligned}
\left\{-\frac{\omega}{c} \epsilon_{1} E_{1} \pm k_{3}^{0 e} H_{2}=0, E_{2}=0, H_{1}=0\right\} & \text { for } k_{3}^{0 e}, \\
\left\{\frac{\omega}{c} \mu_{1} H_{1} \pm k_{3}^{0 p} E_{2}=0, H_{2}=0, E_{1}=0\right\} & \text { for } k_{3}^{0 p},
\end{aligned}
$$

which place them in mutually orthogonal polarizations. The superscripts $p$ and $e$ are used in anticipation of the propagating and exponential nature of these modes, which will be valid within an appropriate frequency band in which $k_{3}^{0 e}=i\left|k_{3}^{0 e}\right|$ and $k_{3}^{0 p}=\left|k_{3}^{0 p}\right|$.

Let the slab consist of the same material, but rotated by a right angle in the $x y$-plane so that its material tensors $\epsilon^{(1)}$ and $\mu^{(1)}$ are

$$
\epsilon^{(1)}=\left[\begin{array}{ccc}
\epsilon_{2} & 0 & 0 \\
0 & \epsilon_{1} & 0 \\
0 & 0 & 1
\end{array}\right], \quad \mu^{(1)}=\left[\begin{array}{ccc}
\mu_{2} & 0 & 0 \\
0 & \mu_{1} & 0 \\
0 & 0 & 1
\end{array}\right] .
$$

The propagation exponents in this medium are the numbers $\left\{k_{3}^{1 p},-k_{3}^{1 p}, k_{3}^{1 e},-k_{3}^{1 e}\right\}$, given by

$$
k_{3}^{1 p}=\left[\epsilon_{2}\left(\frac{\omega^{2}}{c^{2}} \mu_{1}-k_{1}^{2}\right)\right]^{1 / 2}, \quad k_{3}^{1 e}=\left[\mu_{2}\left(\frac{\omega^{2}}{c^{2}} \epsilon_{1}-k_{1}^{2}\right)\right]^{1 / 2},
$$

and their associate eigenspaces are given by the relations

$$
\begin{aligned}
\left\{-\frac{\omega}{c} \epsilon_{2} E_{1} \pm k_{3}^{1 p} H_{2}=0, E_{2}=0, H_{1}=0\right\} & \text { for } k_{3}^{1 p}, \\
\left\{\frac{\omega}{c} \mu_{2} H_{1} \pm k_{3}^{1 e} E_{2}=0, H_{2}=0, E_{1}=0\right\} & \text { for } k_{3}^{1 e} .
\end{aligned}
$$


Figure 2 (left) shows the dispersion relations for the propagating modes of the ambient space (superscript 0 ) and the slab (superscript 1) for hypothetical material coefficients. In the frequency interval $I$ indicated in the figure, the modes $0 e$ and $1 e$ are exponential and the modes $0 p$ and $1 p$ are propagating. What is essential for the existence of such an interval of admissible frequencies is that

$$
\max \left\{\epsilon_{1}, \mu_{2}\right\} \leq \min \left\{\epsilon_{2}, \mu_{1}\right\} \quad \text { and } \quad k_{1} \neq 0 . \quad \text { (assuming } k_{2}=0 \text { ) }
$$

Guided modes at embedded frequencies. For frequencies in the interval $I$, the vector span of the ambient exponential modes $(0 e)$ coincides with that of the propagating modes in the slab $(1 p)$. This allows the construction of guided modes by matching evanescent fields outside the slab with oscillatory fields in the slab:

$$
\begin{aligned}
& {\left[\begin{array}{c}
E_{1} \\
E_{2} \\
H_{1} \\
H_{2}
\end{array}\right]=\left[\begin{array}{c}
-k_{3}^{0 e} \\
0 \\
0 \\
\frac{\omega}{c} \epsilon_{1}
\end{array}\right] e^{-i k_{3}^{0 e} z}, \quad z<0, \quad \text { (leftward evanescent) }}
\end{aligned}
$$

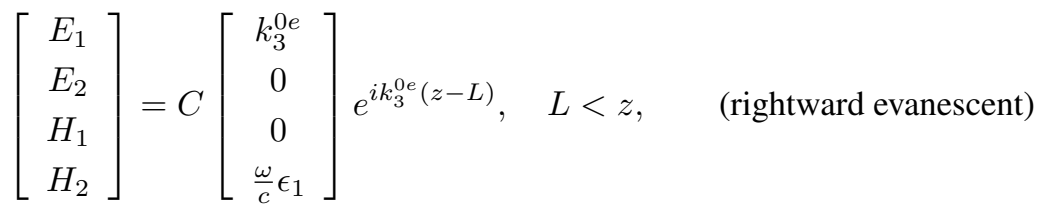

$$
\begin{aligned}
& {\left[\begin{array}{c}
E_{1} \\
E_{2} \\
H_{1} \\
H_{2}
\end{array}\right]=B_{1}\left[\begin{array}{c}
k_{3}^{1 p} \\
0 \\
0 \\
\frac{\omega}{c} \epsilon_{2}
\end{array}\right] e^{i k_{3}^{1 p} z}+B_{2}\left[\begin{array}{c}
-k_{3}^{1 p} \\
0 \\
0 \\
\frac{\omega}{c} \epsilon_{2}
\end{array}\right] e^{-i k_{3}^{1 p} z}, \quad 0<z<L . \quad \text { (oscillatory) }}
\end{aligned}
$$

By imposing the continuity of these solutions at the interfaces $z=0$ and $z=L$, one obtains

$$
2 \cos \left(k_{3}^{1 p} L\right)-i\left(\frac{k_{3}^{0 e}}{k_{3}^{1 p}} \frac{\epsilon_{2}}{\epsilon_{1}}-\frac{k_{3}^{1 p}}{k_{3}^{0 e}} \frac{\epsilon_{1}}{\epsilon_{2}}\right) \sin \left(k_{3}^{1 p} L\right)=0 . \quad \text { (guided-mode condition) }
$$

When plotted in the $\omega-L$ plane, this relation has multiple branches, which are shown in Fig. 2 (middle).
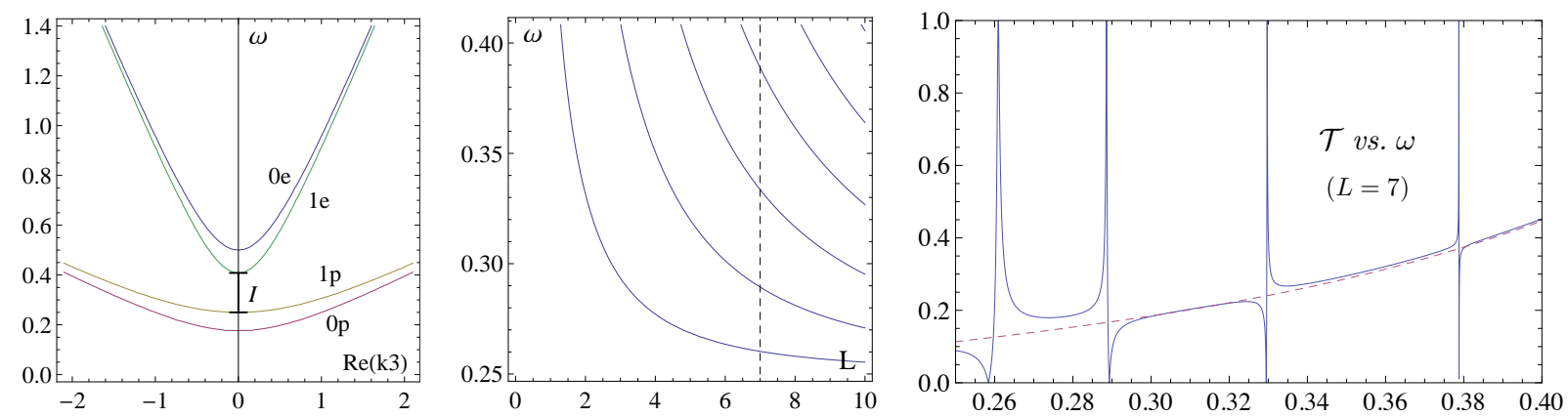

Figure 2. Left: These dispersion relations show frequency $\omega v s$. propagation wavenumber $\operatorname{Re}\left(k_{3}\right)$ perpendicular to the layers ( $z$-direction) for the ambient medium ( $0 p$ and $0 e$ ) and for the slab medium ( $1 p$ and $1 e$ ). For the material coefficients $\epsilon_{1}=1.5, \epsilon_{2}=8, \mu_{1}=4, \mu_{2}=1$, and wavevector $\left(k_{1}, k_{2}\right)=(0.5,0)$ parallel to the layers, there is a frequency interval $I \approx[0.25,0.408248]$ in which each medium admits one propagating mode $(0 p$ and $1 p)$ and one evanescent mode $(0 e$ and 1e). Middle: When the length of the slab $L$ and the frequency $\omega \in I$ satisfy this multi-branched relation, the slab supports a guided mode that falls off exponentially as $|z| \rightarrow \infty$. Because the ambient medium supports a propagating mode for $\omega \in I$, the frequency of the guided mode is embedded in the continuous spectrum. Right: The transmission coefficient is shown for a slab of length $L=7$ for rotations $\phi=0$ (dotted) and $\phi=0.06$ (solid). The unrotated slab $(\phi=0)$ admits guided modes at four frequencies within the interval $I$ indicated by the middle graph. When the slab is rotated about the $z$-axis by a small angle $\phi=0.06$, sharp transmission anomalies appear near the guided-mode frequencies.

Energy transmission across the slab. Even at parameters that satisfy (2), one can prove that the guided mode is decoupled from incident propagating waves in the sense that the scattering problem possesses a steady-state solution 
(it is not unique - the difference of two solutions is a guided mode). In fact, the transmission coefficient is regular as a function of frequency, exhibiting no anomalous behavior, as depicted by the dotted graph in Fig. 2 (right).

Under a typical perturbation of the system, be it structural or in the angle of incidence, the construction of a bound state is no longer possible because the exponential modes at $z=0$ are no longer mapped by the transfer matrix to exponential modes at $z=L$, and the propagating modes in the ambient medium become involved in the equations. One can view this as manifesting the coupling of the bound state to the continuum of extended states, resulting in the dissolution of the embedded bound-state frequency into the continuous spectrum. This results in resonant scattering behavior including amplitude enhancement and extreme sensitivity of the transmission coefficient to frequency.

The solid graph in Fig. 2 (right) shows the emergence of sharp transmission anomalies at the frequencies of the guided modes when the slab is rotated by a small angle about the $z$-axis $\left(\epsilon^{(1)}\right.$ and $\mu^{(1)}$ are multiplied by a rotation matrix). Similar graphs are observed if one perturbs $k_{2}$ from zero. Rigorous analysis of these anomalies is achieved through a complex-analytic connection between the scattering states and (generalized) guided modes for complex frequencies near the parameters of the bound state [4].

The problem of scattering by a harmonic field $J v_{p}^{+} e^{i k_{3}^{0 p} z}$ emanating from a source at $z=-\infty$ is formulated as follows. Let the eigenvectors associated with the exterior propagation constants $\left\{k_{3}^{0 e},-k_{3}^{0 e}, k_{3}^{0 p},-k_{3}^{0 p}\right\}$ be denoted by $\left\{v_{e}^{+}, v_{e}^{-}, v_{p}^{+}, v_{p}^{-}\right\}$. One seeks a solution $w(z)$ of the Maxwell system (1) of the form

$$
w(z)=\left\{\begin{aligned}
J v_{p}^{+} e^{i k_{3}^{0 p} z}+R_{p} v_{p}^{-} e^{-i k_{3}^{0 p} z}+R_{e} v_{e}^{-} e^{-i k_{3}^{0 e} z}, & (z<0) \\
T_{p} v_{p}^{+} e^{i k_{3}^{0 p}(z-L)}+T_{e} v_{e}^{+} e^{i k_{3}^{0 e}(z-L)} . & (z>L)
\end{aligned}\right.
$$

The transmission coefficient, graphed in Fig. 2, is defined as the energy flux of $w(z)$ (which is independent of $z$ ) divided by the energy flux of the incident field $J v_{p}^{+} e^{i k_{3}^{0 p} z}$, and it is equal to $\mathcal{T}=\left|T_{p} / J\right|^{2}$.

\section{A Unidirectional Ambient Medium}

Suppose now that the ambient medium is lossless and unidirectional, meaning that it admits propagation of energy only in one direction, as described in case (B) on the first page. Assume that, at some frequency $\omega_{0}$ and parallel wavevector $\mathbf{k}_{0 \|}$, the layered ambient medium is (rightward) unidirectional. This means that the transfer matrix $M_{0}$ across one period has an eigenvalue of algebraic multiplicity 3 but geometric multiplicity 1 and a distinct eigenvalue of algebraic multiplicity 1 , with both eigenvalues of unit modulus. Let $M_{0}=\exp \left(i \tilde{A}_{0}\right)$. Since $M_{0}$ is unitary with respect to the energy-flux form $[\cdot, \cdot], \tilde{A}_{0}$ can be chosen to be selfadjoint with respect to this form $[6$, III $§ 4.5]$. The linear algebra of indefinite forms [3, Thm. 5.1.1, Cor. 5.2.1] guarantees that there is a basis $\mathcal{B}$ of $\mathbb{C}^{4}$ with respect to which $\tilde{A}_{0}$ is represented by a matrix $A_{0}$ and the energy-flux form $[\cdot, \cdot]$ is represented by a matrix $H$ via $\left[\mathcal{F}_{1}, \mathcal{F}_{2}\right]=v_{1}^{*} H v_{2}$, where the vectors $v_{1,2}$ represent the fields $\mathcal{F}_{1,2}$ in the basis $\mathcal{B}$ and

$$
A_{0}=\left[\begin{array}{cccc}
\alpha & 0 & 0 & 0 \\
0 & \beta & 1 & 0 \\
0 & 0 & \beta & 1 \\
0 & 0 & 0 & \beta
\end{array}\right], \quad H=\left[\begin{array}{cccc}
1 & 0 & 0 & 0 \\
0 & 0 & 0 & -1 \\
0 & 0 & -1 & 0 \\
0 & -1 & 0 & 0
\end{array}\right] .
$$

A generic perturbation of the system (structurally or in frequency) will cause the eigenvalue $\beta$ to split into three simple ones. Suppose that the transfer matrix $M(\eta)$ depends analytically on a parameter $\eta$ (with $\left.M(0)=M_{0}\right)$ so that $A_{0}$ is a member of a one-parameter analytic $H$-selfadjoint family of matrices $A(\eta)$ :

$$
A(\eta)=A_{0}+\eta A_{1}+\eta^{2} A_{2}+\cdots .
$$

The eigenvalues and eigenvectors of $A(\eta)$ for those associated with $\beta$ admit Puiseux series near $\eta=0$, and the first-order terms in their perturbation expansions are nonzero multiples of $a_{42}^{1 / 3} \eta^{1 / 3}$, where $a_{42}$ is the 42 -entry of $A_{1}$. All other entries of the matrices $A_{i}, i \geq 1$, contribute higher-order corrections [5]. In this communication, we will assume in our calculations that $A_{i}=0$ for $i \geq 2$ and that all entries of $A_{1}$ vanish except its 42 -entry, which we set equal to 1 . Thus $A_{0}$ is perturbed by $\eta$ in its entry enclosed in the box in (3). We will show the calculations for $\eta>0$; the case of negative $\eta$ gives in essence the same results.

The four eigenvalues $\lambda_{i}$ of $A(\eta)$ and their eigenvectors $v_{i}$ are

$$
\begin{aligned}
\left\{\lambda_{0}, \lambda_{1}, \lambda_{2}, \lambda_{3}\right\} & =\left\{\alpha, \beta+\eta^{1 / 3}, \beta+\eta^{1 / 3} \zeta, \beta+\eta^{1 / 3} \zeta^{2}\right\} \\
\left\{v_{0}, v_{1}, v_{2}, v_{3}\right\} & =\left\{[1,0,0,0]^{T},\left[0,1, \eta^{1 / 3}, \eta^{2 / 3}\right]^{T},\left[0,1, \eta^{1 / 3} \zeta, \eta^{2 / 3} \zeta^{2}\right]^{T},\left[0,1, \eta^{1 / 3} \zeta^{2}, \eta^{2 / 3} \zeta\right]^{T}\right\},
\end{aligned}
$$


in which $\zeta=(-1+i \sqrt{3}) / 2$ is a primitive cube root of 1 . According to the Floquet theory, for $0<\eta \ll 1$, the vector functions $F(z) w_{i}(z)$, with $w_{i}(z)=v_{i} e^{i \lambda_{i} z}$, form a basis of solutions, where $F(z)$ is an $H$-unitary periodic matrix [6]. With respect to these solutions, the energy-flux form has interaction matrix that is independent of $z$ :

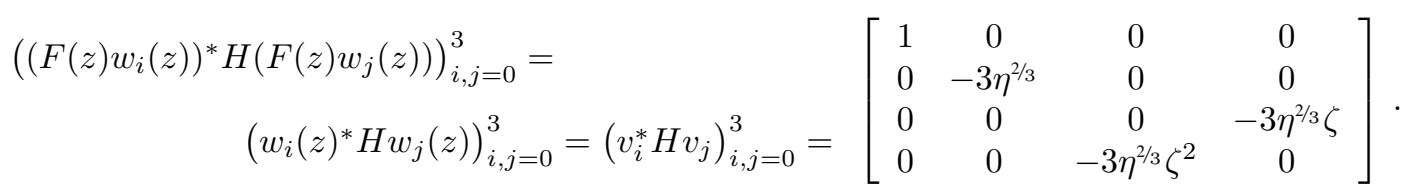

Based on the signs of the self-energy fluxes of $w_{0}(z)$ and $w_{1}(z)$ and the fact that $w_{2}(z)$ is exponentially decaying and $w_{3}(z)$ exponentially growing in $z$, one can designate these solutions as rightward or leftward modes for nonzero $\eta$ :

$$
\begin{array}{rlll}
\text { rightward: } & w_{0}(z)=v_{0} e^{i \alpha z} & \text { and } & w_{2}(z)=v_{2} e^{i\left(\beta+\eta^{1 / 3} \zeta\right) z}, \\
\text { leftward: } & w_{1}(z)=v_{1} e^{i\left(\beta+\eta^{1 / 3}\right) z} & \text { and } & w_{3}(z)=v_{3} e^{i\left(\beta+\eta^{1 / 3} \zeta^{2}\right) z} .
\end{array}
$$

It is only the mode $w_{0}(z)$ that retains its rightward (and propagating) nature in the limit as $\eta \rightarrow 0$. The other modes all tend to the single Bloch mode corresponding to the eigenvector $[0,1,0,0]$ that exists at $\eta=0$, and this mode has vanishing energy flux. Thus the medium becomes unidirectional.

Now let this medium be interrupted by a lossless slab occupying the $z$-interval $[0, L]$. Interesting scattering behavior in the $\eta \rightarrow 0$ limit occurs when one allows the propagating mode $w_{0}(z)$ to be a source field incident upon the slab from the left. The scattering problem for nonzero $\eta$ is to determine coefficients $r_{1}, r_{3}, t_{0}$, and $t_{2}$ such that the Maxwell system admits a solution $w(z)$ of the form

$$
w(z)=F(z) \begin{cases}w_{0}(z)+r_{1} w_{1}(z)+r_{3} w_{3}(z), & z<0 \\ t_{0} w_{0}(z-L)+t_{2} w_{2}(z-L), & z>L .\end{cases}
$$

The transfer matrix $T$ for the slab takes the values of a solution $w(z)$ from $z=0$ to $z=L$ and is unitary with respect to the indefinite energy-flux form:

$$
T w(0)=w(L), \quad T^{*} H=H T^{-1} .
$$

By assuming that $\eta$ represents structural perturbation in the ambient medium and does not involve the frequency or wavenumber $\mathbf{k}_{\|}$, we may allow $T$ to be independent of $\eta$. Assume also that $L$ is a multiple of the period of the ambient medium so that one may take $F(0)=F(L)=I_{4}$ (the identity $4 \times 4$ matrix). The first of equations (5) applied to the solution (4) yields a linear system for the coefficients whose solution is

$$
r_{1}=-\frac{r+O\left(\eta^{1 / 3}\right)}{\eta^{1 / 3}}, \quad r_{3}=\frac{r+O\left(\eta^{1 / 3}\right)}{\eta^{1 / 3}}, \quad t_{0}=\frac{s_{42}}{s+O\left(\eta^{1 / 3}\right)}, \quad t_{2}=-\frac{s_{41}}{s+O\left(\eta^{1 / 3}\right)},
$$

in which $s_{i j}$ is the $i j$-entry of $T^{-1}, s=s_{11} s_{42}-s_{12} s_{41}$, and $r=\left(s_{31} s_{42}-s_{32} s_{41}\right) /\left(s\left(\zeta^{2}-1\right)\right)$.

Nonresonant scattering occurs when the determinants in the definitions of $r$ and $s$ do not vanish. The field on the right of the slab $(z>L)$ exhibits regular behavior, as the coefficients $t_{i}$ are bounded as $\eta \rightarrow 0$. On the left $(z<0)$, although the coefficients $r_{i}$ become unbounded as $\eta \rightarrow 0$, the field $w(z)$ itself remains bounded at any point in the structure because of cancellation of leading-order terms of order $O\left(\eta^{-1 / 3}\right)$ in the second components of the fields $r_{1} w_{1}(z)$ and $r_{3} w_{3}(z)$ :

$$
w(z) \underset{\eta \rightarrow 0}{\longrightarrow} F(z)\left[\begin{array}{c}
e^{i \alpha z} \\
\left.r i\left(\zeta^{2}-1\right) z+c\right) e^{i \beta z} \\
r\left(\zeta^{2}-1\right) e^{i \beta z} \\
0
\end{array}\right] \text { for } z<0, \quad w(z) \underset{\eta \rightarrow 0}{\longrightarrow} \frac{1}{s} F(z)\left[\begin{array}{c}
s_{42} e^{i \alpha(z-L)} \\
-s_{41} e^{i \beta(z-L)} \\
0 \\
0
\end{array}\right] \text { for } z>L
$$

where $c$ is a constant. The cancellation results in the excitation of the second-order normal mode $[0, i z, 1,0] e^{i \beta z}$ possessing linear growth as $z \rightarrow-\infty$. The normal mode involves the second-order root vector of the matrix $A_{0}$ (the third component) corresponding to the eigenvalue $\beta$, whose contribution to the eigenvectors $v_{i}$ vanishes as $\eta \rightarrow 0$. A similar phenomenon occurs when an incident plane wave in vacuum is reflected from the interface with a unidirectional medium [2]. 
Resonant scattering can occur if $s$ or $r$ vanishes. For the transfer matrix $T$ given by

$$
T=\left[\begin{array}{cccc}
1+i & 0 & 1 & 0 \\
0 & 1 & 0 & 0 \\
1 & 0 & 1-i & 0 \\
0 & 0 & 0 & 1
\end{array}\right], \quad T^{-1}=\left[\begin{array}{cccc}
1-i & 0 & -1 & 0 \\
0 & 1 & 0 & 0 \\
-1 & 0 & 1+i & 0 \\
0 & 0 & 0 & 1
\end{array}\right]
$$

both $s$ and $r$ vanish. When $\eta=0$, the slab admits a nonzero field $w^{\text {res }}(z)$ in the absence of any source, namely

$$
w^{\mathrm{res}}(z)=F(z)\left[\begin{array}{l}
0 \\
1 \\
0 \\
0
\end{array}\right] \times \begin{cases}e^{i \beta z}, & z<0 \\
e^{i \beta(z-L)}, & z>L .\end{cases}
$$

This field is the analog of the guided mode of Section I, and it resonates with the rightward-propagating field $F(z)[1,0,0,0]^{T} e^{i \alpha z}$. This is manifest in the solution of the scattering problem (4), which exhibits amplitude enhancement in the transmitted and reflected fields which do not mutually cancel (the components of order $\eta^{-1 / 3}$ ):

$$
w(z)=F(z)\left\{\begin{array}{r}
{\left[\begin{array}{l}
1 \\
0 \\
0 \\
0
\end{array}\right] e^{i \alpha z}-\frac{1}{3-2 i}\left[\begin{array}{c}
0 \\
\eta^{-1 / 3} \\
1 \\
\eta^{1 / 3}
\end{array}\right] e^{i\left(\beta+\eta^{1 / 3}\right) z}-\frac{\zeta}{3-2 i}\left[\begin{array}{c}
0 \\
\eta^{-1 / 3} \\
\zeta^{2} \\
\eta^{1 / 3} \zeta
\end{array}\right] e^{i\left(\beta+\eta^{1 / 3} \zeta^{2}\right) z}, \quad z<0,} \\
\frac{3+i}{3-2 i}\left[\begin{array}{l}
1 \\
0 \\
0 \\
0
\end{array}\right] e^{i \alpha(z-L)}+\frac{\zeta^{2}}{3-2 i}\left[\begin{array}{c}
0 \\
\eta^{-1 / 3} \\
\zeta \\
\eta^{1 / 3} \zeta^{2}
\end{array}\right] e^{i\left(\beta+\eta^{1 / 3} \zeta\right)(z-L)}, \quad z>L .
\end{array}\right.
$$

If the incident field is scaled by $\eta^{1 / 3}$, the total field tends to a multiple of $w^{\text {res }}(z)$ (note that $\left.1+\zeta+\zeta^{2}=0\right)$ :

$$
\lim _{\eta \rightarrow 0} \eta^{1 / 3} w(z)=\frac{\zeta^{2}}{3-2 i} w^{\text {res }}(z) .
$$

\section{DISCUSSION}

Each of the two resonant systems discussed features a source-free field associated with a pole of a scattering matrix at real $\omega$ and $\mathbf{k}_{\|}$, where the scattering problem is not uniquely solvable. This field must satisfy an outgoing but nonradiating (zero energy flux) condition to the left and right of the slab. In case (A), it is the slab that permits matching of evanescent (outgoing) fields. In case (B), the ambient space admits a mode that is spatially extended yet has vanishing energy flux across the layers, and the slab permits matching of this (outgoing) mode to itself. The simultaneous presence of a propagating mode in the ambient space in each case makes these source-free fields unstable with respect to perturbations of the system, and this is the origin of resonant scattering behavior. Analysis of the unidirectional resonance problem is delicate and merits further investigation.

\section{REFERENCES}

[1] Dwight W. Berreman, Optics in stratified and anisotropic media: 4×4-matrix formulation, J. Opt. Soc. Am., vol. 62(4), p. 502-510, 1972.

[2] Alexander Figotin and Ilya Vitebskiy, Electromagnetic unidirectionality in magnetic photonic crystals, Phys. Rev. B, vol. 67, p. 165210-1-20, 2003.

[3] Israel Gohberg, Peter Lancaster, and Leiba Rodman, Indefinite Linear Algebra and Applications, Birkhäuser Verlag, AG, 2005.

[4] Stephen P. Shipman and Stephanos Venakides, Resonant transmission near non-robust periodic slab modes, Phys. Rev. E, vol. 71(1), p. 026611-1-10, 2005.

[5] Aaron Welters, On explicit recursive formulas in the spectral perturbation analysis of a Jordan block, SIAM J. Matrix Anal. Appl., vol. 32(1), p. 1-22, 2011.

[6] V. A. Yakubovich and V. M. Starzhinskii, Linear Differential Equations with Periodic Coefficients I, Halsted Press, 1975. 